\title{
O psicólogo escolar como psicometrista: 30 anos depois
}

\section{Psicometrista}

\author{
Geraldina Porto Witter
}

\section{Resumo}

O objetivo do artigo foi apresentar parte de uma tese não publicada, produzida em 1977, sobre a atuação do psicólogo escolar como psicometrista. Primeiro há uma contextualização sócio-cultural e educacional do documento. Em segundo lugar aparece, em itálico, o documento original com suas referências e na última parte há considerações sobre as mudanças e contrastes que ocorreram com o tempo. A apresentação do documento é concernente às condições acadêmicas e sócio-culturais de sua produção. $O$ texto original é somente um dos papéis do psicólogo escolar apresentados na tese. A última parte apresenta variáveis e novas direções relativas ao psicometrista como evidências para tornar clara a evolução na área. Palavras-chave: história, avaliação psicológica, formação do psicólogo, atuação do psicólogo.

\section{School psychologists psychometrist: 30 years later}

\begin{abstract}
The aim of the paper was to present part of one dissertation did not published, produced at 1977, concerning the school psychologist actuation as psychometrist. First there are a socio-cultural and educational contextualization of the document. In second place appear the original text in italic with it's references and in the last part the are considerations about the changes and contrast that occurred with the time. The presentation of the document is concerning the academic and socio cultural conditions of it production. The original text is only one of the roles of the school psychologist presented in the dissertation. The last part presented variables and new directions concerning the psychometricts as evidences to make clear the evolution in the area. Keywords: history, psychological evaluation, psychologist formation, psychologist actuation.
\end{abstract}

\section{El psicólogo escolar como psicometrista: 30 años después}

\section{Resumen}

El objetivo de este artículo fue presentar parte de una tesis no publicada producida en 1977 sobre la actuación del psicólogo escolar como psicometrista. Primero hay una presentación de un contexto socio-cultural y educacional del documento. En segundo lugar aparece, en itálico, el documento original con sus referencias, y en la última parte hay consideraciones sobre los cambios y contrastes que ocurrieron con el tiempo. La presentación del documento se refiere a las condiciones académicas y socio-culturales de su producción. El texto original es apenas uno de los papeles del psicólogo escolar presentados en la tesis. La última parte presenta variables y nuevos caminos que se refieren al psicometrista, como evidencias para tornar clara la evolución en el área. Palabras clave: historia, evaluación psicológica, formación del psicólogo, actuación del psicólogo. 


\section{Apresentação}

O conhecimento científico vem crescendo em ritmo acelerado desde o século passado. Em conseqüência as mudanças são rápidas, teorias e modelos são substituídos prontamente por novas proposições, parâmetros e indicadores de desenvolvimento tornam-se cada vez mais exigentes. A ciência é imprescindível no cotidiano da vida humana. O ritmo varia de uma para outra ciência e entre as áreas de uma mesma ciência. Ciências mais avançadas chegam a dobrar o conhecimento em um ano ou menos, outras evoluem bem mais lentamente. De qualquer forma, o saber-fazer-poder da ciência torna-se obsoleto cada vez mais rapidamente. Via de regra, se há avanços significativos, espera-se que pelo menos a cada quatro anos, grandes mudanças ocorram e o saber passado seja história e base para a produção atual.

Uma produção com 30 anos de vida certamente é história, mas nem todas as áreas caminham no ritmo acelerado de hoje. O que ainda é válido é tecer comparações, uma forma de avaliar o ritmo em que se está evoluindo. $O$ presente artigo retoma um texto não publicado, que já tem 30 anos e que pode servir de base para se considerar o hoje e o amanhã.

Trata-se de pequena parte da primeira tese de livre-docência defendida no Instituto de Psicologia, criado com a reforma da Universidade de São Paulo. Witter (1977) defendeu uma tese apresentada em três volumes $(839$ p.) sendo que em parte do primeiro deles elaborou um referencial tendo por base os papéis e funções do psicólogo escolar, destacando-se o psicólogo escolar como profissional, clínico, psicometrista, avaliador, consultor, especialista educacional, ergonomista, modificador de comportamento, pesquisador, atuando dentro e fora do sistema educacional.

A Banca Examinadora do processo de livredocência foi constituído pelos professores doutores Maria José de Barros Fornari de Aguirre, Ruy Galvão de Andrada Coelho, Cidmar Teodoro Pais, Odette Lourenção van Kolck, José Pastore e Arrigo Leonardo Angelini que a presidiu,
Dentre os temas tratados foram destacadas para o presente trabalho as páginas sobre o psicólogo escolar psicometrista. Elas seguem transcritas da tese em itálico (Witter, 1977, p. 37-45) mantendo-se o texto sem qualquer interferência de forma e conteúdo. Para tanto, manteve-se o padrão de reprodução de textos históricos, espaçamento e normas vigentes à época da produção. $A$ única interferência foi passar tudo para itálico para indicar sua condição no presente texto. Em seguida são apresentados comentários sobre o que mudou no decorrer de 30 anos.

\section{O psicólogo escolar como psicometrista}

Nos primórdios da Psicologia Escolar, já se definiu o papel de psicometrista como um dos que deveria ser desempenhado pelo profissional que atua na área. Como ocorreu com o papel de Clínico, em alguns países, este passou a ser praticamente o único papel por ele desempenhado, vindo, muitas vezes, ampliado pela adoção também do papel de Clínico. Trata-se de um papel, que, embora possa subsistir por si mesmo, freqüentemente aparece sendo desempenhado como apoio a outros papéis (Roberts, 1970).

Como Psicometrista o Psicólogo Escolar ocupase com a aplicação de testes, notadamente de nível intelectual, visando ao encaminhamento para classes especiais, ou a organização em composição das classes', bem como, as de possibilidade, para atender às necessidades de orientação (vocacional e profissional) e de triagem para a psicoterapia (a ser conduzida na escola ou fora dela).

Como ocorreu com o papel de Psicólogo Escolar Clínico, também o de Psicometrista vem sendo objeto de intensas críticas nos últimos 10 ou 15 anos. Fairchild (1975) critica os papéis tradicionalmente desempenhados pelo psicólogo escolar como psicometrista e clínico para os programas de educação especial, que levaram à crítica e ao próprio questionamento dos serviços de Psicologia na escola. $\mathrm{O}$ autor sugere a mudança de papéis e também um

Veja-se a propósito: Borg (1966). 
cuidadoso registro das próprias atividades diárias, como forma de levantamento de dados que justifiquem sua atuação na escola.

Há um aspecto ligado ao surgimento da Psicologia Escolar como área diferenciada que pode ter contribuído, por um lado para a manutenção quase exclusiva de alguns papéis, por muito mais tempo do que o desejável, e por outro, pela confusão e indefinição de outros. Ligando-se por origem à clínica ou à psicometria (depende do país) e atuando na escola teve, fatalmente, a marcá-la toda a indecisão e falta de delimitação que caracteriza a fusão de campos do conhecimento. As origens marcaram duas funções, a de clínico e a de psicometrista. Esta imagem se concretizou na expectativa do grande público e dos outros profissionais da escola.

Como a Psicologia Escolar representa uma área de transvariação de conhecimentos oriundos de muitas áreas, era de se esperar o que ocorreu, no início, tanto de sua instituição na escola como na formação dos profissionais que se encarregariam de pô-la em prática.

No que tange à aplicação da Psicologia, segundo Bardon (1968), quando se difundiu que através da Psicologia Escolar se pretendia por em prática tudo o que a Psicologia tinha a oferecer à escola, esta transferência foi tentada de uma forma caótica e nada científica. Não se transpôs os cuidados científicos de teste criterioso, e de avaliação cuidadosa da aplicação feita. O resultado lastimável deste fato foi que os psicólogos passaram a atuar ao sabor de suas próprias convicções ou acompanhando o que era moda. A situação era pouco clara mesmo para os próprios Psicólogos Escolares, aos quais faltava identidade profissional, não tinham um conhecimento preciso de seus direitos e eram hipersensíveis quanto ao que os outros esperavam deles. Nestas circunstâncias, por muito tempo e muitas vezes eles se dedicaram mais a fazer o que os outros queriam do que aquilo que poderiam fazer melhor e que traria maiores benefícios para a escola. Estas considerações podem servir para esclarecer a longa insistência nos dois papéis iniciais: clínico e psicometrista.
Muitas das críticas retomam as posições e ponderações feitas em relação ao papel de Psicólogo Clínico, especialmente no que diz respeito ao não atendimento das necessidades mais prementes da escola e do sistema educacional. Estas críticas se aplicam especialmente a sua atividade de seleção e triagem de crianças com problemas. Mesmo quando as críticas são mais suaves, elas concluem pela necessidade de se reduzir o tempo consumido com a aplicação de testes, quer pata liberar o psicólogo para papéis mais úteis, quer para não perturbar o próprio desenvolvimento do processo educacional.

A redução da ênfase do papel de psicometrista cedendo lugar a outros que o Psicólogo Escolar pode exercer reflete, pelo menos em parte, o que vem ocorrendo na história destes instrumentos de avaliação, em parte o desapontamento dos psicólogos e do público com relação aos mesmos.

Poder-se-ia, à guisa de exemplo, lembrar aqui as asserções de Albee em relação a um dos testes mais pesquisados $^{2}$ - o Rorschach, considerado uma das grandes aquisições da Psicologia, com uma validade e credibilidade amplamente difundida, requerendo um longo e difícil treino antes do psicólogo estar apto a aplicá-lo.

Seus poderes eram tão grandes que todo tipo de controle tinha que, ser colocado no preparo daqueles que usariam. Há 20 anos, muitos de nós acreditavam na arte do Rorschach. O público foi fascinado pela mágica que possuíamos. Outras profissões viam nossa mágica com respeito. Mas, então vieram os cientistas cautelosos, a mensuração das pessoas, com suas questões sobre fidedignidade e validade, com suas técnicas sofisticadas, e suas exigências de demonstração pública do valor de nossa mágica sob condições estritamente controladas. Como conseqüência, o Rporschach está quase desaparecendo do cenário da psicologia profissional devido a nossa sensibilidade ao chamado da ciência. (Albee, 1970, p. 1075).

Cleveland estreitamente controladas. Como conseqüência, o Rorschach está quase desaparecendo do cenário da psicologia profissional devido a nossa (1976) arrola uma série de fatores que contribuíram

${ }^{2}$ Ver Reybolds e Sundberg (1976) 
para o declínio do uso de testes. Alguns deles são decorrentes de mudanças nos valores sociais, por natureza bastante difusos, enquanto que outros são mais definitivos e, assim sendo, mais fáceis de identificar. Entre os principais fatores arrolados pelo autor estão os resultados negativos que as pesquisas recentes vêm encontrando; a grande quantidade de estudos mostrando a baixa confiabilidade dos testes; a inadequação e a dificuldade de acordo entre juízes nas interpretações dos testes; a inadequação e $\circ$ baixo nível de precisão; o pouco uso efetivo dos testes em situação de clínica e estando os mesmos mais a satisfazer as necessidades de realização acadêmica do que de trabalho ${ }^{3}$.

Segundo Cleveland, outro fator relevante para o descrédito dos testes reside na crescente sofisticação dos sujeitos face à popularização, às vezes direta às vezes indireta dos mesmos através dos meios de comunicação. Direta quando divulgam suas bases teóricas e científicas. Nestas circunstâncias, aos poucos, os testes acabam por não medir o comportamento real dos sujeitos mas sim aquilo que ele quer que $\circ$ psicólogo pense sobre ele, uma vez que pode responder de acordo com critérios de avaliação e dando de si mesmo a imagem que queira dar ${ }^{4}$.

O terceiro fator importante arrolado com o responsável pela crescente desilusão no uso dos testes encontra-se no seu emprego dentro de um vacum clínico, como se cada um deles por si mesmo, pudesse ser instrumento seguro de diagnóstico e terapia. Outra variável está em íntima relação com esta, tratase da crescente inovação na psicopatologia em termos de modelos teóricos, taxonomia e técnicas psicoterápicas. Outro aspecto a considerar neste descrédito é o alto custo operacional que um trabaIho de aplicação de testes apresenta quando bem feito, implicando no emprego concomitante de vários instrumentos e em muitas horas de trabalho, por vezes desproporcional ao emprego que posteriormente se poderá fazer da informação levantada na prática profissional.

Outra variável apontada por Cleveland (1976) é que os cursos de testes parecem ter decaído de ní- vel, com jovens inexperientes lecionando em lugares dante ocupados por membros muito experientes do quadro universitário. Considera que as faculdades deveriam cuidar para contar com pessoas experientes neste setor uma vez que, mal formados, os futuros psicólogos tenderão a fazer mau uso destes instrumentos e mais desilusão e descrédito eles terão.

Finalmente apresenta como fato contribuindo para - negativismo em relação aos testes a própria idade dos mesmos (Rorschach - 53 anos, TAT - 43 anos, MMPI - 35 anos, WAIS - 19 anos) sem que se tenham levado em conta adequadamente às muitas mudanças sócio-culturais e educacionais pelas quais 0 mundo passou, neste período, nos vários aspectos do material, das instruções, da aplicação e da avaliação dos instrumentos. Seu prognóstico para progresso destes instrumentos implica em mudança no tipo e nas características dos novos testes em desenvolvimento e a serem criados no futuro, com ênfase em escalas de observação do comportamento e em mediadas de aspectos específicos do mesmo, mais do que em instrumentos genéricos abrangendo, por exemplo, toda a "personalidade".

O autor conclui dizendo que o declínio dos testes parece estar diretamente relacionado com o medo de descobrir que os indivíduos diferem. Mudanças nos valores sociais como estas podem ter um grande impacto na mudança nas práticas de psicodiagnóstico. (Cleveland, 1976, p. 317).

Nestas circunstâncias, não é de estranhar a redução do papel de psicometrista e o menor uso de testes como recurso no desempenho de seus papéis. Conseqüentemente, isto deve ter implicações para a revisão da formação destes profissionais.

Muitos destes problemas com os testes e que se refletem no papel de Psicometrista, poderiam ter sido evitados se um maior esforço de pesquisa fosse devotado aos mesmos, antes de se fazer um uso generalizado e indiscriminado dos mesmos. Aqui, o Psicólogo Escolar poderia fornecer uma contribuição significativa não só na produção, mas também na padronização e em outros aspectos relacionados à pesquisa de validade e precisão destes instrumentos.

\footnotetext{
${ }^{4}$ O autor critica inclusive publicações sobre o assunto em revistas como Psychology Today, no Reader's Digest e livros como os de Whyte Jr. (1956) e Black (I962). ${ }^{3}$ Ver também: Meehl (1959); Sines (1970)
} 
Realmente as origens da Psicologia Escolar de certa forma constituíram fatores marcantes no delineamento de alguns papéis atribuídos aos Psicólogos Escolares, conforme já se disse aqui. Mas, ainda que possam parecer incongruentes estes fatores também contribuíram para a diversificação de papéis e técnicas que se registraria mais tarde.

Apoiando-se ora na Psicologia, ora na Educação, ora na Sociologia ou outras disciplinas de área correlata, o Psicólogo Escolar ficou menos restrito que outros especialistas e teve a oportunidade "sui generis" de poder testar novas técnicas, idéias, métodos e instrumentos. Muitos dos quais transpostos de outras áreas que não a Psicologia.

A Psicologia Escolar parece ser a única especialidade na psicologia que considerou seriamente, em todas as épocas, quase todas as práticas correntes na psicologia, na educação, e em campos próximos... Se houver algo a ser tentado o psicólogo escolar o tentará! (Bardon, 1976, p. 787).

Este fato parece ter levado à caracterização de novos papéis e funções para o psicólogo e ampliado sensivelmente a expectativa da escola e da comunidade em relação a este profissional.

\section{Referências}

ALBEE, G.W. The uncertain future of clinical psychology. American Psychologist, 1970, 25: 107I-1080.

BARDON, J.I. School psychology and school psychologists: Are approach to an old problem. The American Psychologist, 1968, 23: 187-194.

BARDON, J.I. School psychology training trends in the early 1970. Professional Psychology, 1976, 7: 31-37.

BLACK, H. They shall not pass. New York: Randon House, 1961.

BORG, W. R. Some fallacies in the "fallacy of homogeneity". Journal of School Psychology, 1966, 5:68-70.

CLEVELAND, S.E. Reflexions on the rise and fall of psychodiagnosis. Professional Psychology, 1976, 7:309-318.

FAIRCHILD, T.N. Accountability: practical suggestions for school psychologists. Journal of School Psychology. 1975, 13: 149-159.
FAIRCHILD, T.N. An analysis of the services performed by a school psychologist in an urban area: Implications for training programs. Psychology in the Schools, 1974, I I: 275-28I.

MEEHL, P.E. A comparison of clinicians with five statistical methods of identifying psychotic MMPI profiles. Journal of Counseling Psychology, 1959, 6: 102-109.

REYNOLDS, W.M. \& SUNDBERG, N.D. Recent research trends in testing. Journal of Personality Assessment, 1976, 40: 228-233.

ROBERTS, R.D. Perceptions of actual and desired role functions of school psychologists by psychologists and teachers, Psychology in the Schools, 1970, 7: 175- 178.

SINES, J.O. Actuarial vs clinical prediction in psychopathology. The British Journal of Psychiatry, 1970, I 16: I 29- 144.

WHYTE JR., W.H. The organization man. New York: Simon \& Schister, 1956.

\section{Comentários}

Conforme se deduz da consulta a obras gerais, como o livro The World of Psychology (Wood \& Wood, 1996) a preocupação com a medida é uma constaste em todas as áreas da Psicologia embora, como no passado, o desenvolvimento de instrumentos específicos se faça mais presente em umas do que em outras. Entre as primeiras e mais freqüentes podem ser mencionadas: memória, inteligência, cognição, linguagem, criatividade, desenvolvimento, aprendizagem, motivação, sexualidade e sócio-psicopatologias.

Medida e instrumentação são partes essenciais das ciências. Em todas elas há sempre um grupo de profissionais dedicados exclusiva ou parcialmente ao seu desenvolvimento. O mesmo ocorre na Psicologia de um modo geral. Todavia, há momentos na história em que tais esforços são mais valorizados ou são vistos como de menor relevância. A tese destaca o clima menos favorável que ocorria nos anos setenta e hoje há uma postura mais positiva, otimista e condizente com as necessidades de uma ciência em desenvolvimento.

A expressão que se desenvolveu com o tempo para designar a área tornou-se mais abrangente e passou a 
incluir os testes foi Avaliação Psicológica, passando no Brasil por uma fase denominada Técnicas de Exame Psicológico (TEP) com que aparece ainda hoje na maioria das grades curriculares dos cursos. Assim hoje é mais raro se usar psicometria embora possa aparecer em textos de história, quando os autores tomam o cuidado metodológico correto de preservar a nomenclatura de época, ou em textos específicos, em geral de cunho metodológico. O texto aqui comentado usa linguagem da época e aponta para as diferenças, (na tese há uma outra parte para Avaliação Witter, 1977), dificuldades e limitações.

Entretanto, ainda é mantida a psicometria que passou a ser considerada como ramo da psicologia dedicada ao desenvolvimento e à aplicação de técnicas estatísticas para análise de dados. Também é mensuração psicológica em que se obtém estimativas numéricas de um aspecto do desempenho. (Walker Jr., 1997). Inclui como sub-áreas: medidas, testes psicológicos, teoria dos itens de resposta, psicofísica, análise estatística, aplicação e interpretação de testes, bem como estudos experimentais envolvendo estes aspectos.

Ainda não está disponível um contexto ideal, adequado, não abusivo dos testes e outras medidas psicológicas, mas a evolução já permite antever progresso substancial na área. $O$ uso exclusivo de testes para avaliação, ainda que tenham boas características psicométricas, continua a ser considerado inadequado por um amplo conjunto de razões como: restrição ou cegueira teórica na visão do que é avaliado, desconstextualização tempo-espacial, abrangência de aspectos do fenômeno estudado, formatação, rápidas mudanças sócio-culturais de hoje que levam à rápida desatualização que as pesquisas de atualização não estão acompanhando no mesmo ritmo, etc. (Fiene, McMahon, 2007, Johnston, Costello, 2005).

No Brasil do começo do século $X X$ até o final do mesmo (anos 90) cresceu a preocupação com a avaliação conforme dados de Alchieri e Scheffer (2000) analisando revistas nacionais com predomínio de testes de Personalidade, seguida de áreas pulverizadas, avaliação intelectual e de habilidades e aptidões. Sendo apreciável o crescimento nas últimas décadas. Noronha e Alchieri (2002) enfatizam a necessidade de pesquisas sobre o desenvolvimento da área no Brasil e de pesquisas de metaciência. A própria estrutura do livro organizado por Primi (2002) mostra a preocupação maior centrada nas áreas tradicionalmente mais investigadas. Também fica registrada alguma preocupação de cunho metodológico.

Em 2003, Pacheco em pesquisa de metaciência enfocando seis periódicos nacionais (1997 a 2002) estudou a produção científica sobre avaliação psicológica verificou que, dos 549 artigos dos periódicos $27,3 \%$ eram sobre o tema pesquisado, sendo que Psicologia: Reflexão e Crítica apresentou maior ocorrência $(33,3 \%)$. Predomina a autoria múltipla o que é um bom resultado em termos de cientometria. No todo houve equilíbrio de gênero entre os produtores, na maioria docentes universitários $(65,8 \%)$, sendo o grupo maior da USP $(18,2 \%)$ e prevalecem as pesquisas $(88,2 \%)$ sobre os trabalhos teóricos ( $11,8 \%)$. Testes e escalas foram os recursos mais estudados (30,1\% e $22,2 \%)$. A produção tende a atender aos parâmetros esperados em ciência como indicativos de bom desenvolvimento.

Para que se estabelecesse condições favoráveis é preciso lembrar algumas variáveis relevantes que ocorreram ao longo do decurso entre a apresentação da tese e a situação atual. Desde a primeira gestão do Conselho Federal de Psicologia, sob a presidência de Arrigo Leonardo Angelini, a preocupação com a avaliação psicológica se fez presente, especialmente com 0 uso inadequado de testes e escalas. Encontros de especialistas, discursos em eventos foram feitos, mas se esbarrava na resistência dos próprios profissionais. Nas sucessivas gestões do Conselho Federal de Psicologia a matéria continuou como uma dificuldade a ser superada. A Resolução CFP 025/200I ao definir os testes psicológicos como método de avaliação privativo do psicólogo e regulamentar sua elaboração, comercialização e uso viabilizou um grande avanço de qualidade na produção científica e no uso de testes e escalas no Brasil.

Também é preciso lembrar que o aumento da produção de testes em condições de uso seguro passou a influir não apenas na qualidade dos serviços, mas na credibilidade das avaliações feitas. Conseqüentemente, melhorou a imagem dos profissionais dedicados à 
área não só na Psicologia como no intercâmbio com outros profissionais.

Este clima mais favorável às medidas tem uma abrangência mundial e decorre de melhor cuidado com aspectos diversos dos testes (Wechsler, Guzzo, 1999), do próprio amadurecimento da Psicologia e dos responsáveis por seu crescimento. Aspectos específicos do processo de criação dos testes passaram a ser melhor cuidados. Apesar de se carecer de estudos profundos sobre os cuidados no uso e interpretação dos resultados, tudo indica que a situação hoje é bem mais compatível com o científica e eticamente recomendável. Todavia, vale atentar para as considerações de Patto (1997) ainda pertinentes quando a formação de profissional não o alerta para problemas como o uso inadequado e político de instrumentos de avaliação. Uma produção científica de qualidade e uma formação sólita evitam erros e usos indevidos.

Um exemplo é o trabalho Núñez, Núñez e Pinha (2006) enfocando técnicas para detectar os padrões aberrantes de respostas que são úteis na construção de testes e para a criação de bancos de itens para testes, com características psicométricas e de validade conhecida. Certamente são conhecimentos importantes tanto para os que produzem como para os que usam tais instrumentos. Em cursos de pós-graduação centrados nos testes, escalas e outras medidas psicológicas é certamente um assunto de relevo já que como lembram os autores a questão diz respeito a todas as principais atividades da Psicometria (escalograma, teorias, generalização, análise de itens, modelos não paramétricos etc).

A credibilidade dos instrumentos também decorreu de desenvolvimento de instrumentos específicos isolados ou de baterias de testes para avaliar questões específicas. Como exemplo de bateria deste tipo pode ser a pesquisada por Sartori, Belliard, Chevier, Trebon e Edan (2006) para avaliar déficit cognitivo decorrente de esclerose múltipla que leva à incapacitação progressiva. A bateria validada em francês compreende vários testes, detecta sinais diversos, viabiliza encaminhamentos, o acompanhamento do tratamento e a eficácia potencial de vários procedimentos terapêuticos. Como exemplo do primeiro caso pode-se lembrar a Construção da Escala de Empregabilidade: competências e habilidades sociais, elaborada por Lara Campos (2006) que fez um amplo e profundo estudo psicométrico, propondo instrumento de que tanto era carente a Psicologia no Brasil, quer no setor organizacional, quer no de orientação profissional.

Também nas últimas décadas, os movimentos de inclusão das pessoas atípicas ou com necessidades especiais impulsionou parte da psicometria para 0 desenvolvimento de instrumentos específicos para diversas limitações físicas. Cromwell (2005) retoma as questões enfrentadas pelos psicólogos para testar pessoas que apresentam sérias limitações auditivas. $O$ uso da linguagem de sinais foi um avanço, mas nem todo psicometrista ou pessoa surda domina a linguagem. Espera que a Arte da Psicometria resolva tais problemas. Crítica o comodismo dos psicometristas e dos profissionais da área que parecem acomodados no contexto de ignorância do qual precisam sair e evoluir. Muitos parecem não estar cientes de mudanças específicas em Psicometria. Mas outros estão criando novas possibilidades.

Entretanto, apesar dos avanços alcançados há ainda um longo caminho a percorrer para melhorar a área da psicometria não só no Brasil como no exterior. Ainda há carência de instrumentos específicos em muitas áreas. Bedford (2006) lembra a necessidade da psicometria disponibilizar instrumentos para o diagnóstico de demência, por exemplo.

$\mathrm{Na}$ área da Psicologia Escolar, o papel da psicometria, segundo Damusi e Desjarlais (2004) continua a ter destaque na medida e descrição das diferenças intelectuais, na definição de políticas e técnicas educacionais, na avaliação de estilos perceptivos cognitivos, modos de percepção, tratamento da informação, dependência - independência de campo, estabilidade psicológica, diferenciação psicológica, gênero, estilo cognitivo, traços de personalidade, mecanismos de defesa, problemas psicopatológicos entre outros. Em uma perspectiva mais sintética as áreas em que mais estão sendo usados os testes, segundo os autores são cognição, aprendizagem de comportamentos típicos dos vários estilos cognitivos, matérias acadêmicas, solução de problemas e apren- 
dizagem de conceitos. Os instrumentos são recursos para fazer avaliações, por exemplo, do estilo cognitivo de modo a facilitar a aprendizagem para os alunos.

A questão de gênero é uma preocupação constante entre educadores e pesquisadores educacionais para garantir equidade no atendimento (McCormick, 2006). Para que tenham igual atenção é preciso medir as diferenças em capacidades cognitivas, atitudes (Cassidy, 2006) o que é básico para educação para a cidadania (Crocco e Libresco, 2006) e para inclusão (Brown e Roy, 2006, Koch, 2006, Bell e Norwood, 2006). Nestas circunstâncias, não é de surpreender o surgimento e o aprimoramento de testes na área.

Pelo exposto é evidente que hoje as condições ambientais e técnico-científicas criaram uma maior aceitabilidade e uso crítico das medidas psicológicas, notadamente para os testes e escalas. Entretanto é necessário conduzir pesquisas junto aos profissionais que estão atuando para uma caracterização do como o recomendável se efetiva. Também é preciso verificar como os futuros psicólogos estão sendo formados no que concerne à avaliação. Certamente inserir umas poucas questões constantes no instrumento de avaliação dos alunos dos cursos, embora útil, não é suficiente. São necessárias pesquisas mais específicas e que enfoquem aspectos conceituais, metodológicos e práticos da aplicação e uso de tais instrumentos.

\section{Referências}

Alchieri, J. C., \& Scheffel, M. (2000). Indicadores da Produção Científica Brasileira em Avaliação Psicológica: resultados da elaboração de uma base de dados dos artigos publicados em periódicos brasileiros de 1930 a 1999. Anais do V Encontro Mineiro de Avaliação Psicológica e prática e VIII Conferência Internacional de Avaliação Psicológica - formas e contextos (pp. 99100). Belo Horizonte: PUC-MINAS.

Bedford, S. (2006). Disseminating psychological skills in old age psychiatry services. Psychiatric Bulletin, 30(5), 194.

Bell, K. N., \& Norwood, K. (2006). Gender Equity Intersets with Mathematics and Technology: problem-solving education for changing times. Em D. Sadker \& E. S. Silber (Eds.), Gender in the classroom: foundations skills, methods, and strategies across the curriculum (pp. 225-258). Condon: Lawrence Erlbaum Ass.
Brown, S. P., \& Roy, P. A. (2006). A Gender - Inclusive Approach to English/ Language Arts Methods: literacy with a critical lens. Em D. Sadker \& Silber, E. S. (Eds.), Gender in the classroom: foundations skills, methods, and strategies across the curriculum (pp. 165-204). Condon: Lawrence Erlbaum Ass.

Cassidy, K. W. (2006). Gender differences in Cognitive Ability, Attitudes, and Behavior. Em D. Sadker \& E. S. Silber (Eds.), Gender in the classroom: foudations, skills, methods, and strategies across the curriculum (pp. 33-72). Condon: Lawrence Erlbaum Ass., Publs.

Crocco, M. S., \& Libresco, A. S. (2006). Citizenship Education for the studies. Em D. Sadker \& E. S. Silber (Eds.), Gender in the classroom: foundations skills, methods, and strategies across the curriculum (pp. 109-164). Condon: Lawrence Erlbaum Ass., Publs.

Cromwell, J. (2005). Deafness and the art of psychometric testing. The Psychologist, I8(12), 738-740.

Damusi, V., \& Desjarlais, L. (2004). La dépendance independance du champ comme style cognitif: certaines consequences psychopedagogiques. European Review of Applied Psychology/ Revue Européenne de Psychologie Appliquée, 54(2), 65-72.

Fiene, J., \& McMahon, S. (2007) Assessing comprehension a classroom-based process. The Reading Teacher, 60(5), 406419.

Johnston, P., \& Costello, P. (2005). Principles for literacy assessment. Reading Research Quarterly, 40(2), 256-267.

Kock, J. (2006). A Gender Inclusive Approach to Science Education. Em D. Sadker \& E.S. Silber (Eds.), Gender in the classroom: foundations skills, methods, and strategies across the curriculum (pp. 205-224). Condon: Lawrence Erlbaum Ass., Publs.

Lara Campos, K. C. (2006). Construção de uma escala de empregabilidade: competências e habilidades pessoais. Tese Doutorado, Instituto de Psicologia, Universidade de São Paulo, São Paulo.

McComick, J. (2006). Strong Women Teachers: their struggles and strategies for gender equity. Em D. Sadker \& E. S. Silber (Eds.), Gender in the classroom: foundations skills, methods, and strategies across the curriculum (pp. 1-32). Condon: Lawrence Erlbaum Ass., Publs.

Noronha, A. P. P., \& Alchieri, J. C. (2002). Reflexões sobre os Instrumentos de avaliação psicológica. Em R. Primi (Org.), 
Temas em Avaliação Psicológica (pp. 7- 16). Campinas: Instituto Brasileiro de Avaliação Psicológica.

Núñez, R. M., Núñez, P., \& Pina, J. A. L. (2006). Técnicas para detectar patrones de respuesta atípicas. Anales de Psicologia, 22(I), 143-154.

Pacheco, E. M. C. (2003). Produção Científica em Avaliação Psicológica: Análise de Periódicos Brasileiros (1997-2002). Tese de Doutorado, Pontifícia Universidade Católica de Campinas, Campinas.

Patto, M. H. S. (1997). Para uma crítica da razão psicométrica. Psicologia - USP, 8(I), 47-62.

Primi, R. (Org.). (2002). Temas em avaliação psicológica. Campinas: Instituto Brasileiro de Avaliação Psicológica.

Sartori, E., Belliard, S., Chevrier, C., Trebon, P., Chaperon, J., \& Edan, G. (2006). De la psychométrie au handicap neuropsychometrique dans la selérose en plaques. Proposition d'une batterie de déspistage en langue française et facteurs de risqué cognitifs. Revue Neurologique, I62(5), 603-6I5.

Walker Jr., A. (Ed.). (1997). Thesaurus of psychological index terms. Washington, DC: American Psychological Association.

Wechsler, S. M., \& Guzzo, R. S. L. (Orgs.). (1999). Avaliação Psicológica: perspectiva internacional. São Paulo: Casa do Psicólogo.

Witter, G. P. (1977). O Psicólogo Escolar: pesquisas e ensino. Tese de Livre Docência em Psicologia Escolar, Instituto de Psicologia, Universidade de São Paulo, São Paulo.

Wood, S. E., \& Wood, E. G. (1996). The world of Psychology $\left(2^{\mathrm{a}}\right.$ ed.). Boston: Allyn and Bacon.

Recebido em 16/04/2007

Revisado em: 08/10/2007

Aprovado em: 17/12/2007

Sobre a autora:

Geraldina Porto Witter (gwitter@uol.com.br) - Universidade Castelo Branco

Av. Pedroso de Moraes, I44/302 - Pinheiros - CEP 05420-000 - São Paulo - SP 